\title{
Octupole deformations in actinides at high spins within the cranking Skyrme-Hartree-Fock approach
}

\author{
A. Tsvetkov ${ }^{1}$, J. Kvasil $^{1}$ and R.G. Nazmitdinov ${ }^{2}$ \\ ${ }^{1}$ Particle and Nuclear Physics Institute, Charles University \\ $V$ Holešovičkách 2, 18000 Praha 8, Czech Republic \\ ${ }^{2}$ Bogoliubov Laboratory of Theoretical Physics, Joint Institute for Nuclear Research \\ 141980 Dubna, Moscow Region, Russia
}

\begin{abstract}
The cranked Skyrme III effective Hamiltonian is applied for the analysis of the rotational dependence of the quadrupole and octupole moments in Ra, Th, and $\mathrm{U}$ isotopes. A comparison of the intrinsic electric dipole moments calculated in the model with available experimental and theoretical values is presented. It is found that the non-axial octupole deformation $Y_{32}$ becomes favorable at high spins for the actinide nuclei.
\end{abstract}

PACS number(s): 21.60-n; 21.60.Jz; 21.10.Ky; 27.90.+b

In the last decade extensive experimental data have been obtained on low-lying states of negative parity (see for review [1.21). For the $R a-T h(Z \sim 88, N \sim 134)$ and $B a-S m(Z \sim 56, N \sim 88)$ nuclei low $3^{-}$states, parity doublets, alternating parity bands with enhanced dipole transitions have been found. Since the octupole interactions are strongest when pairs of orbitals from the intruder sub-shell $(l, j)$ and the normal parity sub-shell $(l-3, j-3)$ are near the Fermi surface, the octupole correlations are expected to become important for these nuclei [3

The features observed in nuclei are very similar to ones familiar from molecular physics. In molecules a stable octupole deformation leads to the appearance of the rotational bands with the alternating parity levels connected by strong E1 intra-band transitions. Möller and Nilsson used the assumption about a symmetry breaking of the intrinsic reflection symmetry of the single-particle potential and demonstrated the instability with respect to the axial octupole deformation in the Ra-Th region [5. The analysis was performed within the macroscopic-microscopic approach that combines the liquid drop model and the Strutinsky shell correction method for a modified harmonic oscillator potential. Later, within this approach numerous results, based on various single-particle phenomelogical potentials, have been reported regarding the presence of a stable axial octupole deformation in the ground state of some nuclei in the actinide region [2]. The macroscopic-microscopic approach was also used in the combination with the cranking model to analyze properties of rotating nuclei with a stable axial octupole deformation (see e.g. [6, []). The problem of non-axial octupole deformations, being for a long time a terra incognita, attracts more attention during last years. The study of the non-axial octupole deformations could shed light on the tendency for the system on the way toward fission to avoid the superdeformation [3]. Calculations by the macroscopic-microscopic method with the Woods-Saxon potential predicted the importance of the banana-type $Y_{31}$ deformation for highly deformed nuclei [8], while the $Y_{32}$ deformation was found to be important in the ${ }^{222} R a$ nucleus [9]. Manifestations of pronounced shell effects have been discovered when non-axial octupole deformations are added to a harmonic oscillator model [10].

Although the macroscopic-microscopic approach is a very efficient practical tool for the analysis of shell effects in finite Fermi systems, basically, it is a phenomenological method which is unable, for example, to predict binding energies. Development of microscopic approaches, which start with the effective nucleon-nucleon interactions treated within a self-consistent Hartree-Fock (HF) method, opened a new avenue in the nuclear structure studies [4, 2]. Using the Skyrme III effective interaction, Bonche et al. [11] did the first self-consistent calculations for the ground states of the Ra-Th region. It was found that the total energy has a minimum for the nonzero value of the $Q_{30}$ momentum in ${ }^{222} R a$. Robledo and Egido 12 employed the Hartree-Fock- Bogoliubov method with the Gogny interaction and found the axial octupole deformation in the ground states in ${ }^{222,224} R a,{ }^{222} \mathrm{Th}$ and ${ }^{142-148} \mathrm{Ba}$. Recent self-consistent HF+Skyrme calculations [13] suggest that the oblate states in nuclei with $A \sim 80$ are soft against the $Y_{33}$ deformation in the ground states. The self-consistent cranking calculations based upon the HF+Skyrme [14] and the HF+Gogny [15] approaches predict that the $Y_{31}$ deformation is important for a correct description of the yrast band of ${ }^{32} S$. However, the systematic investigation of the non-axial octupole deformations in the actinide region at high spins is missing. On the other hand, the calculations in the framework of the $s p d f$ IBM model show that the $1-p f$ boson limit (dipole-octupole vibrations) can describe essential features related to the octupole bands in the Rn-Th region [16]. Using the quasiparticle spectrum generated by the mean field with the reflection symmetry, the quasiparticle-phonon model reproduced quite well the experimental data for $B(E 1)$-transition probabilities in ${ }^{227-231} \mathrm{Ra}$ and ${ }^{229-231} \mathrm{Th}$ [17. As is seen from this discussion, the question of the existence of stable octupole deformations in the actinide region at different angular momenta is still controversial and challenging for microscopic approaches. In this paper the dipole, quadrupole and octupole intrinsic moments of rotating nuclei from the Ra-Th-U region are studied within 
the HF + Skyrme approach. To this aim we exploit the novel version (1.75) of the code HFODD [18] for the Skyrme III force [19] in the particle-hole channel. The code was successfully tested for the analysis of superdeformed nuclei (see, for example, [20]). Therefore, the basis employed by the code is sufficient for our study. In the present calculations the pairing correlations are neglected. They are not expected to play an important role at large rotational frequencies, however, they may influence results obtained for the ground state.

We briefly remind the main ingredients of the calculations performed with the code HFODD, since it will be useful for our discussion below. The code 21 solves the Hartree-Fock problem in rotating frame with the cranking Hamiltonian in the form

$$
H_{\Omega}=T+V+V_{\text {coul }}-\Omega \hat{I}_{y}-\sum_{\tau=n, p} \lambda_{\tau} \hat{N}_{\tau}+\sum_{\lambda \mu} C_{\lambda \mu}\left(\hat{Q}_{\lambda \mu}-\bar{Q}_{\lambda \mu}\right)^{2} .
$$

Here $T$ represents the kinetic energy operator, $V$ is the Skyrme potential, $V_{\text {coul }}$ stands for the Coulomb term. The quantity $\Omega$ is the rotational frequency, $\hat{I}_{y}$ is the total angular momentum projection onto the cranking axis (yaxis); $\lambda_{\tau}$ and $\hat{N}_{\tau}$ are the chemical potential and the particle number operator, respectively, for protons $(\tau=p)$ and neutrons $(\tau=n)$. The last term in (11) represents multipole constraints. It was introduced in order to find a good minimum of the total energy that corresponds to the expected symmetry of the mean field (see below). $\hat{Q}_{\lambda \mu}$ is the mass-multipole-moment operator (with the multipolarity $\lambda$ and the projection $\mu$ ), $\bar{Q}_{\lambda \mu}$ is the expectation value of the corresponding multipole moment for a given rotational frequency $\Omega$. The stiffness parameter $C_{\lambda \mu}$ determines the weight (or our priority) of the constraint for a given $\lambda$ and $\mu$ during the minimization process. The detailed description of each term in (11) can be found in 18,21]. Minimizing the expectation value of the cranking Hamiltonian $H_{\Omega},<H F ; \Omega\left|H_{\Omega}\right| H F ; \Omega>$, for a given rotational frequency $\Omega$ and a fixed set of the Skyrme parameters, the yrast state and equilibrium values of mass-multipoles momenta, $\left\langle H F ; \Omega\left|\hat{Q}_{\lambda \mu}\right| H F ; \Omega>\right.$ can be found. The knowledge of possible symmetries of a searched state could simplify the minimization procedure. We used the following options of the code:

i. The mean field is supposed to be symmetric with respect to the transformations $\hat{S}_{y}=\hat{P} e^{-i \pi \hat{I}_{y}}, \hat{S}_{x}^{T}=\hat{T} \hat{P} e^{-i \pi \hat{I}_{x}}$, $\hat{S}_{z}^{T}=\hat{T} \hat{P} e^{-i \pi \hat{I}_{z}}(\hat{T}$ is the time reversal operator, $\hat{P}$ is the parity operator). Such a situation corresponds to the standard $R$-invariance [3] when the expectation value, $\left\langle H F ; \Omega\left|\hat{Q}_{\lambda \mu}\right| H F ; \Omega>\right.$, is nonzero only for even values of $\lambda$ and $\mu$. These symmetries are characteristic of well-deformed nuclei without any octupole deformations. There are three symmetry planes $(y-z, x-z, x-y)$ of the mean field.

ii. The only mean field symmetry is $\hat{S}_{y}=\hat{P} e^{-i \pi \hat{I}_{y}}$ (there is only one $x-z$ symmetry plane). This is a general case when all expectation values $\left\langle H F ; \Omega\left|\hat{Q}_{\lambda \mu}\right| H F ; \Omega>\right.$ can be nonzero.

In the case $\mathbf{i}$ ) the quantum numbers signature $r= \pm i$ (an eigenvalue of the operator $\hat{R}_{y}=e^{-i \pi \hat{I}_{y}}$ ) and parity $\pi= \pm 1$ can be ascribed to each HF single-particle state. In the case ii) the parity is not a good quantum number and the HF single-particle state can be characterized by the quantum number simplex: $s= \pm i$ (an eigenvalue of the operator $\hat{S}_{y}$ ) (see a discussion about different symmetries in [22]). In each cases discussed above one can reduce the number of iterations in the HF problem by the appropriate constraint value $\bar{Q}_{\lambda \mu}$ in the last term in (1). In addition, the last term in (1) allows to avoid physically meaningless "deformations" which could correspond, for example, to a shift of the nucleus center of mass. In order to ensure the translation invariance of the Hamiltonian we used the constraints with $\bar{Q}_{10}=\bar{Q}_{11}=0$ and the constraint $\bar{Q}_{21}=\bar{Q}_{2-1}=0$ for the principal-axis condition.

The calculations for three chains of isotopes ( $\mathrm{Ra}$, Th, and $\mathrm{U}$ ) were performed without imposing a restriction on the axial reflection symmetry (the option ii)). For each nucleus two minima of the total energy were obtained: one corresponds to nonzero octupole moments and the second minimum is related to the mean field solution with the conserved reflection symmetry (the option i)). In order to obtain the reflection asymmetric minimum the optimal values for the constraint $\bar{Q}_{30}=10^{2} \mathrm{fm}^{3}$ and for the stiffness parameter $C_{30} \approx 0.01$ were found and used in the calculations. The constraints for the other octupole moments result in the smaller binding energies. Both the minima of the total energy are very close. For instance, for a well octupole deformed nucleus ${ }^{226} R a$ the octupole deformed minimum is lower by $1 \mathrm{MeV}$ with respect to the reflection-symmetric minimum. All plots presented on Figs.1-4 are related to the reflection asymmetric minima. From these figures it follows that the octupole deformed Ra, Th, and U isotopes have axial symmetric shapes at small rotational frequencies $\Omega$. With the increase of the rotational frequency both the non-axial moments $Q_{22}$ and $Q_{32}$ increase whilst the axial moments $Q_{20}$ and $Q_{30}$ decrease for all three elements studied. The exceptions are provided by the lightest isotopes of $\mathrm{Ra}$ and $\mathrm{Th}\left({ }^{218-220} \mathrm{Ra},{ }^{218-220} \mathrm{Th}\right)$. In these isotopes the moment $Q_{30}$ reaches the maximum at $\Omega \sim 0.3 \mathrm{MeV}$ and then decreases. Similar tendency regarding the $Q_{30}$ moment has been predicted for even-even rotating isotopes ${ }^{220-228} \mathrm{Th}$ in the macroscopic-microscopic approach [23]. However, in the calculations 23] only axially symmetric shapes of the Woods-Saxon potential have been considered. At 
larger rotational frequencies, we obtained that heavier isotopes, especially, of uranium, behave as non-axial octupole deformed systems. In Fig. 3 the intervals of the rotational frequencies $\Omega$ for which the reflection asymmetric minimum lies below the reflection symmetric one are denoted by arrows. From Fig.4 it follows that the non-axial octupole moment $Q_{32}$ increases with the increase of the rotational frequency in ${ }^{221,225,228} \mathrm{Ra},{ }^{223,229,231} \mathrm{Th}$ and ${ }^{226,231,233-238} \mathrm{U}$ isotopes. This predominance of the non-axial octupole deformation in some nuclei may be tested by measurements of the $E 1$ transitions. A small displacement between the center-of-mass and the center of charge, caused by the non-axial deformation, may enlarge the $E 1$ transitions within a parity doublet. In Fig. 5 the induced electric dipole moment $D_{0}$,

$$
D_{0}=e\left[\frac{N}{A} Q_{10}(p)-\frac{Z}{A} Q_{10}(n)\right]
$$

is shown for different mass numbers $\mathrm{A}$ and different values of the rotational frequency. In (2) the quantities $Q_{10}(p)$ and $Q_{10}(n)$ are the proton and neutron contributions to the mass-dipole-moment $Q_{10}$, respectively. As is seen in Figs. 3 and 5 , the value of $D_{0}$ is correlated with the axial octupole moment $Q_{30}$. The larger the value of $Q_{30}$ the larger is the electric dipole moment $D_{0}$ induced by the octupole deformation. This tendency is consistent with all known results concerning the $D_{0}$ (see [2] and the references there). The non-axial octupole deformation $Q_{32}$ influences the value of $D_{0}$ as well, however, its contribution is less essential. In the chain of isotopes, from the lighter to the heavier one, we obtained the sign change of the quantity $D_{0}$, which preserves at different rotational frequencies. This behavior is similar to the one predicted in [12] with the Gogny forces for Ra and Th isotopes without rotation. A small value of $D_{0}$ preserves to high rotational frequency in ${ }^{222-224} \mathrm{Ra},{ }^{226-228} \mathrm{Th}$ and ${ }^{232,233} \mathrm{U}$. Small changes in the quantity $D_{0}$ over the full frequency range is obtained in ${ }^{222-224} \mathrm{Ra},{ }^{226-228} \mathrm{Th}$ and ${ }^{230-233} \mathrm{U}$. Notice that for ${ }^{222-224} \mathrm{Ra}$ our results are consistent with the experimental observation of the behavior of $D_{0}$ 24]. The noticeable changes in the $D_{0}$ due to the rotation are obtained in ${ }^{228,231,233,234} \mathrm{Ra},{ }^{220,230,231,233,234} \mathrm{Th}$ and ${ }^{223-227} \mathrm{U}$. In Fig. 6 the calculated values of $\left|D_{0}\right|$ are compared with the results of the other approaches and with the experimental values extracted from the $B(E 1)$-transition probabilities [2]. We remind that these experimental data provide only the absolute value $\left|D_{0}\right|$. The comparison is done for two values of the rotational frequency $\Omega=0 \mathrm{MeV}$ (low spin region) and $\Omega=0.1 \mathrm{MeV}$ (high spin region). One can see from Fig. 6 that our results are in agreement with the other calculations and that they reflect a qualitative behavior of the experimental data for Ra and Th isotopes. It would be interesting to test our prediction for $\mathrm{U}$ isotopes within other approaches and by experimental measurements. In our analysis the quantity $D_{0}$ is nonzero and has a physical meaning only in the intervals denoted by the arrows in Fig.3. However, the correlations caused by residual interactions can also contribute to the values of the total energy [25] and multipole moments [26]. Consequently, although the main features should be preserved, the range of the intervals may be modified. The work in this direction is in the progress.

In summary, we analyzed the mass-multipole-moments $Q_{2 \mu}(\mu=0,2), Q_{3 \mu}(\mu=0,1,2,3)$ and the intrinsic electric dipole moment $D_{0}$ in the Ra-Th-U region. The analysis has been done within the self-consistent cranking $+\mathrm{HF}$ approach using the Skyrme III effective interaction. It was found that the octupole moment $Q_{30}$ decreases whilst the moment $Q_{32}$ increases with the increase of the rotational frequency $\Omega$. A similar behavior is found for the quadrupole moments $Q_{20}$ and $Q_{22}$. The quantity $D_{0}$ changes the sign at $\Omega=0 \mathrm{MeV}$ at $A \sim 224$ (for Ra), $A \sim 227$ (for Th), and at $A \sim 232$ (for $\mathrm{U}$ ). This tendency preserves over the whole range of values of the rotational frequency $0.0 \leq \Omega \leq 0.6$ $\mathrm{MeV}$.

\section{Acknowledgments}

Authors would like to express their gratitude to Jacek Dobaczewski for providing the (1.75) version of the HFODD code and for valuable discussions concerning the application of his code. The work was supported in part by a Czech Republic Grant No. 202/99/1718 and by the Russian Foundation for Basic Research under Grant 00-02-17194.

[1] I. Ahmad and P.A. Butler, Annu.Rev.Nucl.Part.Sci. 43, 71 (1993).

[2] P.A. Butler and W. Nazarewicz, Rev. Mod. Phys. 68, 349 (1996).

[3] A. Bohr and B.R. Mottelson, Nuclear Structure (Benjamin, New York, 1975) v.2.

[4] S. Åberg, H. Flocard and W. Nazarewicz, Annu.Rev.Nucl.Part.Sci. 40, 439 (1990).

[5] P. Möller and S.G. Nilsson, Phys. Lett. B31, 283 (1970).

[6] W. Nazarewicz, P. Olanders, I. Ragnarsson, J. Dudek and G. Leander, Phys.Rev.Lett. 52, 1272 (1984).

[7] S.Frauendorf and V.V. Pashkewich, Phys. Lett. B141, 23 (1984). 
[8] R.R. Chasman, Phys.Lett. B266, 243 (1991).

[9] X. Li and J. Dudek, Phys.Rev. C49, R1250 (1994).

[10] W. D. Heiss, R. A. Lynch and R. G. Nazmitdinov, JETP Lett. 69, 563 (1998); Phys. Rev. C60, 034303 (1999).

[11] P. Bonche, P.-H. Heenen, H. Flocard and D. Vautherin, Phys. Lett. B175, 387 (1986).

[12] J.L. Egido and L.M. Robledo, Nucl. Phys. A518, 475 (1990).

[13] S. Takami, K. Yabana and M. Matsuo, Phys. Lett. B431, 242 (1998).

[14] M. Yamagami and K. Matsuyanagi, Nucl.Phys. A672, 123 (2000).

[15] T. Tanaka, R.G. Nazmitdinov and K. Iwasawa, arXiv: nucl-th/0004009.

[16] N.V. Zamfir, private communication and to be published in Yad.Fizika, 2001.

[17] A.J. Aas, H. Mach, J. Kvasil et al, Nucl.Phys. A654, 499 (1999); L.M. Fraile, A.J. Aas, M.J.G. Borge et al, Nucl.Phys. A657, 355 (1999) and references there.

[18] J. Dobaczewski and J. Dudek, Comp. Phys. Comm. 102, 166 (1997); 102, 183 (1997).

[19] M. Beiner, H. Flocard, Nguyen van Giai and P. Quentin, Nucl. Phys.A238, 29 (1975).

[20] J. Dobaczewski and J. Dudek, Phys.Rev. C52, 1827 (1995).

[21] J. Dobaczewski, the 1.75 version of the HFODD code, private communication.

[22] J. Dobaczewski, J. Dudek, S. G. Rohozinski and T. R. Werner, Phys.Rev. C62, 014310 (2000); Phys.Rev. C62, 014311 (2000)

[23] P.A. Butler and W. Nazarewicz, Nucl. Phys. A533, 249 (1991).

[24] J.F.C. Cocks, P.A. Butler, K.J. Cann, et al, Phys.Rev.Lett. 78, 2920 (1997).

[25] F. Dönau, D. Almehed and R.G. Nazmitdinov, Phys.Rev.Lett. 83, 280 (1999).

[26] J.Kvasil and R.G. Nazmitdinov, Czech.J.Phys. 48, 21 (1998).

\section{Figure Capture}

Fig.1 The quadrupole moment $Q_{20}$ as a function of the rotational frequency. The results are displayed: for Raisotopes at the top panel, for Th-isotopes at the middle panel and for U-isotopes at the bottom panel.

Fig.2 The quadrupole moment $Q_{22}$. Similar to the Fig.1

Fig.3 The octupole moment $Q_{30}$. Similar to the Fig.1. See also the text.

Fig.4 The octupole moment $Q_{32}$. Similar to the Fig.1

Fig.5 The electric dipole moment $D_{0}$ induced by the octupole deformation as a function of the rotational frequency. Similar to the Fig.1

Fig.6 Experimental (with error bars) and calculated intrinsic dipole moment $\left|D_{0}\right|$. Experimental and calculated values are indicated by symbols denoted: "els" and "tls" for measurements and our results for low-spin states $(I \sim 0 \hbar)$, respectively; "ehs" and "ths" for measurements and our results for higher spin states $(I \sim 8 \hbar)$, respectively. Experimental values are taken from [2]. The symbols "BNls" and "BNhs" correspond to the results from [23] obtained at $\Omega=0 \mathrm{MeV}$ and $\Omega=0.1 \mathrm{MeV}$, respectively. The calculated values denoted by "ER" are taken from [12]. 
This figure "fig1.gif" is available in "gif" format from: http://arxiv.org/ps/nucl-th/0009085v2 
This figure "fig2.gif" is available in "gif" format from: http://arxiv.org/ps/nucl-th/0009085v2 
This figure "fig3.gif" is available in "gif" format from: http://arxiv.org/ps/nucl-th/0009085v2 
This figure "fig4.gif" is available in "gif" format from: http://arxiv.org/ps/nucl-th/0009085v2 
This figure "fig5.gif" is available in "gif" format from: http://arxiv.org/ps/nucl-th/0009085v2 
This figure "fig6.gif" is available in "gif" format from: http://arxiv.org/ps/nucl-th/0009085v2 